\title{
Leptogenesis and dark matter from a low scale seesaw mechanism
}

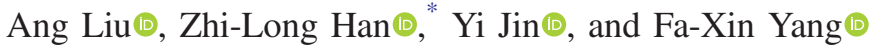 \\ School of Physics and Technology, University of Jinan, Jinan, Shandong 250022, China
}

(Received 15 January 2020; accepted 20 April 2020; published 6 May 2020)

\begin{abstract}
In this paper, we perform a detailed analysis of the phenomenology of leptogenesis and dark matter from a low scale seesaw mechanism. In the framework of Neutrinophilic two Higgs doublet model, we further introduce one scalar singlet $\phi$ and one Dirac fermion singlet $\chi$, which are charged under a $Z_{2}$ symmetry. Assuming the coupling of $\chi$ is extremely small, it serves as feebly interacting massive particle (FIMP) dark matter. Heavy right-hand neutrinos $N$ provide a common origin for tiny neutrino mass (via a seesaw mechanism), leptogenesis (via $N \rightarrow \ell_{L} \Phi_{\nu}^{*}, \bar{\ell}_{L} \Phi_{\nu}$ ), and dark matter (via $N \rightarrow \chi \phi$ ). With hierarchical righthand neutrino masses, the explicit calculation shows that successful thermal leptogenesis is viable even for TeV-scale $N_{1}$ with $0.4 \mathrm{GeV} \lesssim v_{\nu} \lesssim 1 \mathrm{GeV}$ and lightest neutrino mass $m_{1} \lesssim 10^{-11} \mathrm{eV}$. In such a scenario, light FIMP dark matter in the $\mathrm{keV}$ to $\mathrm{MeV}$ range is naturally expected. The common parameter space for neutrino mass, natural leptogenesis, and FIMP dark matter is also obtained in this paper.
\end{abstract}

DOI: 10.1103/PhysRevD.101.095005

\section{INTRODUCTION}

Besides the success of the standard model (SM), there are still several open questions. In particular, the tiny neutrino mass, baryon asymmetry of the Universe (BAU), and dark matter (DM) are the three outstanding pieces of evidence that require physics beyond the SM. The discovery of neutrino oscillations [1,2] indicates that the masses of neutrinos are at the sub-eV scale, which is at least 6 orders of magnitude smaller than the charged leptons. Known as the type-I seesaw mechanism $[3,4]$, this extensively considered way to naturally incorporate neutrino masses is via introducing three right-hand neutrinos $N$ together with high scale Majorana masses of $N$,

$$
-\mathcal{L}_{Y} \supset y \bar{L} \tilde{\Phi} N+\frac{1}{2} \bar{N}^{c} m_{N} N+\text { H.c. },
$$

where $\Phi$ is the SM Higgs doublet. After spontaneous electroweak symmetry breaking, neutrinos achieve masses as

$$
m_{\nu}=-\frac{v^{2}}{2} y m_{N}^{-1} y^{T}
$$

Typically, $m_{\nu} \sim \mathcal{O}(0.1) \mathrm{eV}$ is obtained with $y \sim \mathcal{O}(1)$ and $m_{N} \sim \mathcal{O}\left(10^{14}\right) \mathrm{GeV}$. Meanwhile, the heavy neutrino can also account for BAU via leptogenesis [5]. For canonical thermal

\footnotetext{
*sps_hanzl@ujn.edu.cn
}

Published by the American Physical Society under the terms of the Creative Commons Attribution 4.0 International license. Further distribution of this work must maintain attribution to the author(s) and the published article's title, journal citation, and DOI. Funded by SCOAP ${ }^{3}$. leptogenesis with hierarchal right-hand neutrinos, an upper limit on the charge-parity $(C P)$ asymmetry exists; thus, a lower limit on the mass of lightest right-hand neutrino $M_{1}$ should be satisfied [6],

$$
M_{1} \gtrsim 5 \times 10^{8} \mathrm{GeV}\left(\frac{v}{246 \mathrm{GeV}}\right)^{2} .
$$

Therefore, both tiny neutrino mass and leptogenesis favor the high scale $N$ in the type-I seesaw mechanism. However, for such a high scale $N$, a naturalness problem might arise [7]. By requiring radiative corrections to the $m_{\Phi}^{2} \Phi^{\dagger} \Phi$ term no larger than $1 \mathrm{TeV}^{2}$, it is found that [8]

$$
M_{1} \lesssim 3 \times 10^{7} \mathrm{GeV}\left(\frac{v}{246 \mathrm{GeV}}\right)^{2 / 3}
$$

should be satisfied. It is clear that naturalness is incompatible with canonical leptogenesis. One viable pathway to overcome this is lowering the leptogenesis scale by imposing resonant leptogenesis [9], an Akhmedov-Rubakov-Smirnov mechanism via neutrino oscillation $[10,11]$, or Higgs decay $[12,13]$. The success of these scenarios depends on the degenerate mass of right-hand neutrinos $[14,15]$, which seems is another sense of unnaturalness. An alternative scenario with hierarchal right-hand neutrinos is employing an intrinsic low scale neutrino mass model, e.g., Neutrinophilic two Higgs doublet model ( 2 2HDM) [16,17] or Scotogenic model [18-26]. In this paper, we consider $\nu 2 \mathrm{HDM}$ [27]. Based on the previous brief discussion in Refs. [17,28,29], we perform a detailed analysis of leptogenesis, especially focusing on dealing with the corresponding Boltzmann equations to obtain the viable parameter space. 
On the other hand, dark matter accounts for more than 5 times the proportion of visible baryonic matter in our current cosmic material field. In principle, one can regard the lightest right-hand neutrino $N_{1}$ at the $\mathrm{keV}$ scale as sterile neutrino DM [30-33]. However, various constraints leave a quite small viable parameter space [34]. Meanwhile, leptogenesis with two hierarchal right-hand neutrinos is still at high scale $[23,35,36]$. In this paper, we further introduce a dark sector with one scalar singlet $\phi$ and one Dirac fermion singlet $\chi$, which are charged under a $Z_{2}$ symmetry [37,38]. Stability of DM $\chi$ is protected by the $Z_{2}$ symmetry; therefore, the stringent $\mathrm{x}$-ray limits can be avoided [34]. In light of the null results from DM direct detection [39] and indirect detection [40], we consider $\chi$ as feebly interacting massive particle (FIMP) DM [41].

The structure of the paper is as follows. In Sec. II, we briefly introduce our model. Leptogenesis with hierarchal right-hand neutrinos is discussed in Sec. III. The relic abundance of FIMP DM $\chi$ and constraint of free-streaming length are considered in Sec. IV. Viable parameter space for leptogenesis and DM is obtained by a random scan in Sec. V. We conclude our work in Sec. VI.

\section{THE MODEL}

The original $\mathrm{TeV}$-scale $\nu 2 \mathrm{HDM}$ for neutrino mass was proposed in Ref. [27]. The model is extended by one neutrinophilic scalar doublet $\Phi_{\nu}$ with the same quantum numbers as the SM Higgs doublet $\Phi$ and three right-hand heavy neutrinos $N$. To forbid the direct type-I seesaw interaction $\bar{L} \tilde{\Phi} N$, a global $U(1)_{L}$ symmetry should be employed, under which $L_{\Phi}=0, L_{\Phi_{\nu}}=-1$, and $L_{N}=0$. Therefore, $\Phi_{\nu}$ will specifically couple to $N$, and $\Phi$ will couple to quarks and charged leptons as in the SM. For the dark sector, one scalar singlet $\phi$ and one Dirac fermion singlet $\chi$ are further introduced, which are charged under a $Z_{2}$ symmetry. Provided $m_{\chi}<m_{\phi}$, then $\chi$ serves as a DM candidate.

The scalar doublets could be denoted as

$$
\Phi=\left(\begin{array}{c}
\phi^{+} \\
\frac{v+\phi^{0, r}+i \phi^{0, i}}{\sqrt{2}}
\end{array}\right), \quad \Phi_{\nu}=\left(\begin{array}{c}
\phi_{\nu}^{+} \\
\frac{v_{\nu}+\phi_{\nu}^{0, r}+i \phi_{\nu}^{0, i}}{\sqrt{2}}
\end{array}\right) .
$$

The corresponding Higgs potential is then

$$
\begin{aligned}
V= & m_{\Phi}^{2} \Phi^{\dagger} \Phi+m_{\Phi_{\nu}}^{2} \Phi_{\nu}^{\dagger} \Phi_{\nu}+m_{\phi}^{2} \phi^{\dagger} \phi+\frac{\lambda_{1}}{2}\left(\Phi^{\dagger} \Phi\right)^{2} \\
& +\frac{\lambda_{2}}{2}\left(\Phi_{\nu}^{\dagger} \Phi_{\nu}\right)^{2}+\lambda_{3}\left(\Phi^{\dagger} \Phi\right)\left(\Phi_{\nu}^{\dagger} \Phi_{\nu}\right)+\lambda_{4}\left(\Phi^{\dagger} \Phi_{\nu}\right)\left(\Phi_{\nu}^{\dagger} \Phi\right) \\
& -\left(\mu^{2} \Phi^{\dagger} \Phi_{\nu}+\text { H.c. }\right) \\
& +\frac{\lambda_{5}}{2}\left(\phi^{\dagger} \phi\right)^{2}+\lambda_{6}\left(\phi^{\dagger} \phi\right)\left(\Phi^{\dagger} \Phi\right)+\lambda_{7}\left(\phi^{\dagger} \phi\right)\left(\Phi_{\nu}^{\dagger} \Phi_{\nu}\right),
\end{aligned}
$$

where the $U(1)_{L}$ symmetry is broken explicitly but softly by the $\mu^{2}$ term. For the unbroken $Z_{2}$ symmetry, $\langle\phi\rangle=0$ should be satisfied. Meanwhile, vacuum expectation values (VEVs) of Higgs doublets in terms of parameters of the Higgs potential can be found by deriving the minimization condition

$$
\begin{gathered}
v\left[m_{\Phi}^{2}+\frac{\lambda_{1}}{2} v^{2}+\frac{\lambda_{3}+\lambda_{4}}{2} v_{\nu}^{2}\right]-\mu^{2} v_{\nu}=0, \\
v_{\nu}\left[m_{\Phi_{\nu}}^{2}+\frac{\lambda_{2}}{2} v_{\nu}^{2}+\frac{\lambda_{3}+\lambda_{4}}{2} v^{2}\right]-\mu^{2} v=0 .
\end{gathered}
$$

Taking the parameter set

$$
m_{\Phi}^{2}<0, \quad m_{\Phi_{\nu}}^{2}>0, \quad\left|\mu^{2}\right| \ll m_{\Phi_{\nu}}^{2},
$$

we can obtain the relations of VEVs as

$$
v \simeq \sqrt{\frac{-2 m_{\Phi}^{2}}{\lambda_{1}}}, \quad v_{\nu} \simeq \frac{\mu^{2} v}{m_{\Phi_{\nu}}^{2}+\left(\lambda_{3}+\lambda_{4}\right) v^{2} / 2} .
$$

Typically, $v_{\nu} \sim 1 \mathrm{GeV}$ is obtained with $\mu \sim 10 \mathrm{GeV}$ and $m_{\Phi_{\nu}} \sim 100 \mathrm{GeV}$. Since the $\mu^{2}$ term is the only source of $U(1)_{L}$ breaking, radiative corrections to $\mu^{2}$ are proportional to $\mu^{2}$ itself and are only logarithmically sensitive to the cutoff [42]. Thus, VEV hierarchy $v_{\nu} \ll v$ is stable against radiative corrections $[43,44]$.

After spontaneous symmetry breaking, the physical Higgs bosons are given by [45]

$H^{+}=\phi_{\nu}^{+} \cos \beta-\phi^{+} \sin \beta, \quad A=\phi_{\nu}^{0, i} \cos \beta-\phi^{0, i} \sin \beta$,

$H=\phi_{\nu}^{0, r} \cos \alpha-\phi^{0, r} \sin \alpha, \quad h=\phi^{0, r} \cos \alpha+\phi^{0, r} \sin \alpha$,

where the mixing angles $\beta$ and $\alpha$ are determined by

$\tan \beta=\frac{v_{\nu}}{v}, \quad \tan 2 \alpha \simeq 2 \frac{v_{\nu}}{v} \frac{-\mu^{2}+\left(\lambda_{3}+\lambda_{4}\right) v v_{\nu}}{-\mu^{2}+\lambda_{1} v v_{\nu}}$.

Neglecting the terms of $\mathcal{O}\left(v_{\nu}^{2}\right)$ and $\mathcal{O}\left(\mu^{2}\right)$, the masses of the physical Higgs bosons are

$$
\begin{aligned}
m_{H^{+}}^{2} & \simeq m_{\Phi_{\nu}}^{2}+\frac{1}{2} \lambda_{3} v^{2}, \quad m_{A}^{2} \simeq m_{H}^{2} \simeq m_{H^{+}}^{2}+\frac{1}{2} \lambda_{4} v^{2}, \\
m_{h}^{2} & \simeq \lambda_{1} v^{2} .
\end{aligned}
$$

Since the mixing angles are suppressed by the small value of $v_{\nu}, h$ is almost identical to the $125 \mathrm{GeV}$ SM Higgs boson 
[46,47]. A degenerate mass spectrum of $\Phi_{\nu}$ as $m_{H^{+}}=$ $m_{H}=m_{A}=m_{\Phi_{\nu}}$ is adopted in our following discussion for simplicity, which is certainly allowed by various constraints [48]. Because of the unbroken $Z_{2}$ symmetry, the dark scalar singlet $\phi$ does not mix with the Higgs doublets.

The new Yukawa interaction and mass terms are

$-\mathcal{L}_{Y} \supset y \bar{L} \tilde{\Phi}_{\nu} N+\lambda \bar{\chi} \phi N+\frac{1}{2} \overline{N^{c}} m_{N} N+m_{\chi} \bar{\chi} \chi+$ H.c., where $\tilde{\Phi}_{\nu}=i \sigma_{2} \Phi_{\nu}^{*}$. Similar to the canonical type-I seesaw mechanism [3], the mass matrix for light neutrinos can be derived from Eq. (16) as

$$
m_{\nu}=-\frac{v_{\nu}^{2}}{2} y m_{N}^{-1} y^{T}=U_{\mathrm{PMNS}} \hat{m}_{\nu} U_{\mathrm{PMNS}}^{T}
$$

where $\hat{m}_{\nu}=\operatorname{diag}\left(m_{1}, m_{2}, m_{3}\right)$ is the diagonalizable neutrino mass matrix, and $U_{\text {PMNS }}$ is the PMNS (PontecorvoMaki-Nakagawa-Sakata) matrix:

$$
U_{\mathrm{PMNS}}=\left(\begin{array}{ccc}
c_{12} c_{13} & s_{12} c_{13} & s_{13} e^{i \delta} \\
-s_{12} c_{23}-c_{12} s_{23} s_{13} e^{-i \delta} & c_{12} c_{23}-s_{12} s_{23} s_{13} e^{-i \delta} & s_{23} c_{13} \\
s_{12} s_{23}-c_{12} c_{23} s_{13} e^{-i \delta} & -c_{12} s_{23}-s_{12} c_{23} s_{13} e^{-i \delta} & c_{23} c_{13}
\end{array}\right) \times \operatorname{diag}\left(e^{i \varphi_{1} / 2}, 1, e^{i \varphi_{2} / 2}\right)
$$

Here, we use abbreviations $c_{i j}=\cos \theta_{i j}$ and $s_{i j}=\sin \theta_{i j}, \delta$ is the Dirac phase, and $\varphi_{1}, \varphi_{2}$ are the two Majorana phases. Because of the smallness of $v_{\nu}$, TeV-scale $m_{N}$ could be viable to realize $0.1 \mathrm{eV}$ scale light neutrino masses. Using the Casas-Ibarra parametrization [49,50], the Yukawa matrix $y$ can be expressed in terms of neutrino oscillation parameters

$$
y=\frac{\sqrt{2}}{v_{\nu}} U_{\mathrm{PMNS}} \hat{m}_{\nu}^{1 / 2} R\left(\hat{m}_{N}\right)^{1 / 2},
$$

where $R$ is an orthogonal matrix in general and $\hat{m}_{N}=$ $\operatorname{diag}\left(M_{1}, M_{2}, M_{3}\right)$ is the diagonalizable heavy neutrino mass matrix. In this work, we parametrize matrix $R$ as

$$
\begin{aligned}
R= & \left(\begin{array}{ccc}
\cos \omega_{12} & -\sin \omega_{12} & 0 \\
\sin \omega_{12} & \cos \omega_{12} & 0 \\
0 & 0 & 1
\end{array}\right)\left(\begin{array}{ccc}
\cos \omega_{13} & 0 & -\sin \omega_{13} \\
0 & 1 & 0 \\
\sin \omega_{13} & 0 & \cos \omega_{13}
\end{array}\right) \\
& \times\left(\begin{array}{ccc}
1 & 0 & 0 \\
0 & \cos \omega_{23} & -\sin \omega_{23} \\
0 & \sin \omega_{23} & \cos \omega_{23}
\end{array}\right)
\end{aligned}
$$

where $\omega_{12,13,23}$ are arbitrary complex angles.

\section{LEPTOGENESIS}

Now we consider the leptogenesis of this model. The lepton asymmetry is generated by out-of-equilibrium $C P$-violating decays of right-hand neutrino $N \rightarrow \ell_{L} \Phi_{\nu}^{*}$, $\bar{\ell}_{L} \Phi_{\nu}$. Neglecting the flavor effect [51], the $C P$ asymmetry is given by

$$
\epsilon_{i}=\frac{1}{8 \pi\left(y^{\dagger} y\right)_{i i}} \sum_{j \neq i} \operatorname{Im}\left[\left(y^{\dagger} y\right)_{i j}^{2}\right] G\left(\frac{M_{j}^{2}}{M_{i}^{2}}, \frac{m_{\Phi_{\nu}}^{2}}{M_{i}^{2}}\right),
$$

where the function $G(x, y)$ is defined as [36]

$G(x, y)=\sqrt{x}\left[\frac{(1-y)^{2}}{1-x}+1+\frac{1-2 y+x}{\left(1-y^{2}\right)^{2}} \ln \left(\frac{x-y^{2}}{1-2 y+x}\right)\right]$.

Using the parametrization of Yukawa coupling $y$ in Eq. (19), it is easy to verify

$$
y^{\dagger} y=\frac{2}{v_{\nu}^{2}} \hat{m}_{N}^{1 / 2} R^{\dagger} \hat{m}_{\nu} R \hat{m}_{N}^{1 / 2}
$$

Hence, the matrix $y^{\dagger} y$ does not depend on the PMNS matrix, which means that the complex matrix $R$ is the source of $C P$ asymmetry $\epsilon_{i}$. The asymmetry is dominantly generated by the decay of $N_{1}$. Further considering the hierarchal mass spectrum $m_{\Phi_{\nu}}^{2} \ll M_{1}^{2} \ll M_{2,3}^{2}$, the asymmetry $\epsilon_{1}$ is simplified to

$$
\epsilon_{1} \simeq-\frac{3}{16 \pi\left(y^{\dagger} y\right)_{11}} \sum_{j=2,3} \operatorname{Im}\left[\left(y^{\dagger} y\right)_{1 j}^{2}\right] \frac{M_{1}}{M_{j}} .
$$

Similar to the Davidson-Ibarra bound [6], an upper limit on $\epsilon_{1}$ can be derived

$$
\left|\epsilon_{1}\right| \lesssim \frac{3}{16 \pi} \frac{M_{1} m_{3}}{v_{\nu}^{2}}
$$

Comparing with the bound in the type-I seesaw mechanism, the asymmetry could be enhanced due to the smallness of VEV $v_{\nu}$. Therefore, low scale leptogenesis seems to be viable in the $\nu 2 \mathrm{HDM}[17,28]$. Meanwhile, the washout effect is quantified by the decay parameter 


$$
K=\frac{\Gamma_{1}}{H(z=1)}
$$

where $\Gamma_{1}$ is the decay width of $N_{1}, H$ is the Hubble parameter, and $z \equiv M_{1} / T$ with $T$ being the temperature of the thermal bath. The decay width is given by

$$
\Gamma_{1}=\frac{M_{1}}{8 \pi}\left(y^{\dagger} y\right)_{11}\left(1-\frac{m_{\Phi_{\nu}}^{2}}{M_{1}^{2}}\right)^{2},
$$

and the Hubble parameter is

$$
H=\sqrt{\frac{8 \pi^{3} g_{*}}{90}} \frac{T^{2}}{M_{p l}}=H(z=1) \frac{1}{z^{2}},
$$

with $g_{*}$ the effective number of relativistic degrees of freedom and $M_{p l}=1.2 \times 10^{19} \mathrm{GeV}$. Using Eq. (23), one can verify

$$
K \simeq 897\left(\frac{v}{v_{\nu}}\right)^{2} \frac{\left(\hat{m}_{\nu}^{R}\right)_{11}}{\mathrm{eV}},
$$

where $\hat{m}_{\nu}^{R} \equiv R^{\dagger} \hat{m}_{\nu} R$, and thus,

$$
\begin{aligned}
\left(\hat{m}_{\nu}^{R}\right)_{11}= & m_{1}\left|\cos \omega_{12}\right|^{2}\left|\cos \omega_{13}\right|^{2}+m_{2}\left|\sin \omega_{12}\right|^{2}\left|\cos \omega_{13}\right|^{2} \\
& +m_{3}\left|\sin \omega_{13}\right|^{2} .
\end{aligned}
$$

It is obvious that the decay parameter $K$ does not depend on $\omega_{23}$, and it is also enhanced by the smallness of $v_{\nu}$. Since $\left(\hat{m}_{\nu}^{R}\right)_{11}$ is typical of the order of $m_{3} \sim 0.1 \mathrm{eV}$, the decay parameter $K \simeq 5.4 \times 10^{6}$ when $v_{\nu}=1 \mathrm{GeV}$. So, even with maximum asymmetry $\epsilon_{1}^{\max } \sim-6.0 \times 10^{-7}$ for $M_{1}=$ $10^{5} \mathrm{GeV}$ obtained from Eq. (25), a rough estimation of

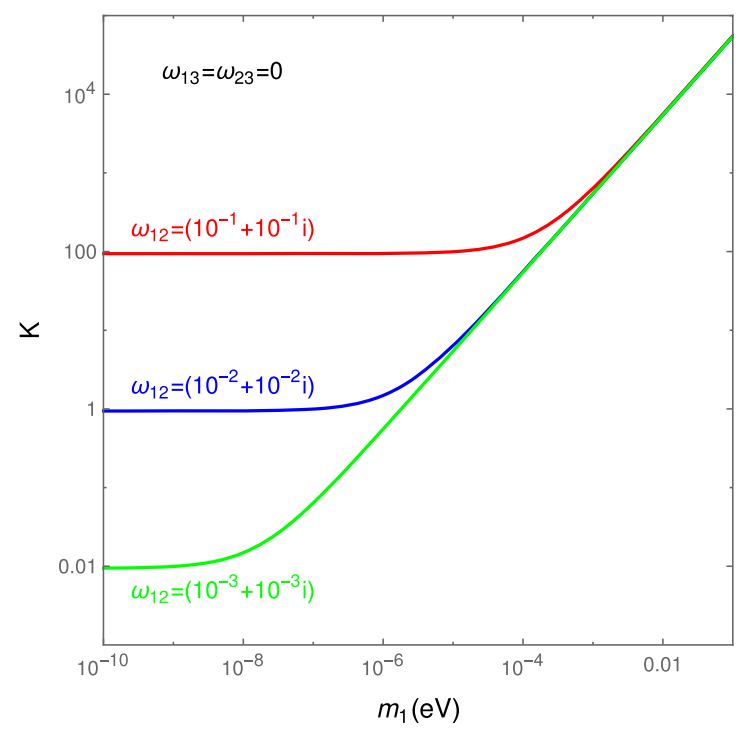

the final baryon asymmetry gives $Y_{\Delta B} \sim-10^{-3} \epsilon_{1}^{\max } / K \sim$ $1.1 \times 10^{-16}$ for strong washout [52], which is far below the currently observed value $Y_{\Delta B}^{\mathrm{obs}}=(8.72 \pm 0.04) \times 10^{-11}$ [53]. Hence, only obtaining an enhanced $C P$ asymmetry $\epsilon_{1}$ is not enough, one has to deal with the washout effect more carefully.

One promising pathway is to reduce the decay parameter $K$. For instance, if the weak washout condition $K \lesssim 1$ is realized, then $Y_{\Delta B} \sim-10^{-3} \epsilon_{1}^{\max } \sim 6.0 \times 10^{-10}>Y_{\Delta B}^{\text {obs }}$. Thus, correct baryon asymmetry can be obtained by slightly tuning $\epsilon_{1}$. As pointed out in Ref. [23], the small value of $K$ can be realized by choosing small $\omega_{12,13}$. In Fig. 1, we illustrate the dependence of $K$ on lightest neutrino mass $m_{1}$ with $v_{\nu}=10 \mathrm{GeV}$. The left panel shows the special case $\omega_{13}=0$, where Eq. (30) is simplified to $\left(\hat{m}_{\nu}^{R}\right)_{11}=$ $m_{1}\left|\cos \omega_{12}\right|^{2}+m_{2}\left|\sin \omega_{12}\right|^{2} \geq \sqrt{\Delta m_{21}^{2}}\left|\sin \omega_{12}\right|^{2}$. It is clear that the weak washout condition $K<1$ favors $\left|\omega_{12}\right| \lesssim 10^{-2}$ and $m_{1} \lesssim 10^{-6} \mathrm{eV}$. The right panel shows the special case $\omega_{12}=0$. Similar results are observed in the left panel.

Since the matrix $R$ has no physical meaning, we further consider the resulting Yukawa couplings of $N$. Then, one can derive an upper bound on the Yukawa couplings of $N_{1}$ from the out-of-equilibrium condition $\Gamma_{1}<H(z=1)$, i.e.,

$$
\left|y_{i 1}\right|^{2}<4 \times 10^{-17} \frac{M_{1}}{1 \mathrm{GeV}} \text {. }
$$

The above tiny Yukawa couplings of $N_{1}$ mean that the dominant contributions to neutrino masses are from $N_{2,3}$. To generate neutrino mass $m_{3} \gtrsim \sqrt{\Delta m_{\mathrm{atm}}^{2}} \simeq 0.05 \mathrm{eV}$, Eq. (19) implies a lower bound on the Yukawa couplings of $N_{2,3}$,

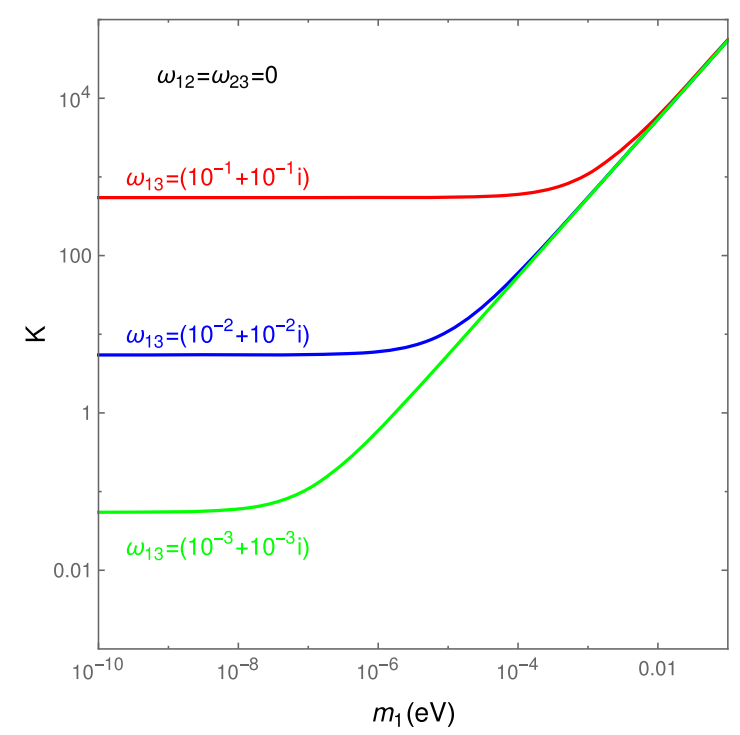

FIG. 1. Decay parameter $K$ as a function of $m_{1}$ with $v_{\nu}=10 \mathrm{GeV}$. Because $R$ must be a complex matrix, we have set $\omega_{i j R}=\omega_{i j I}$. 


$$
y_{i j} \gtrsim \frac{1 \mathrm{GeV}}{v_{\nu}} \sqrt{\frac{M_{j}}{1 \mathrm{GeV}}} \times 10^{-5} \quad(j=2 \text { or } 3) .
$$

Therefore, a hierarchical structure of Yukawa coupling $y$ is needed to induce leptogenesis at low scale for the nondegenerate mass of $N[19,54]$. That is to say, the small value of $\omega_{12}$ or $\omega_{23}$ of matrix $R$ is to realize such a hierarchical structure of $y$. One can naturally achieve the suppression of Yukawa coupling $y_{i 1}$ by imposing additional symmetry. For instance, a $Z_{2}^{\prime}$ symmetry is introduced, under which $N_{1}$ and one new scalar singlet $\xi$ are $Z_{2}^{\prime}$ odd. Then, an effective interaction $y_{\xi} \bar{L} \tilde{\Phi}_{\nu} N_{1} \xi / \Lambda$ is obtained, and $y_{i 1}=y_{\xi}\langle\xi\rangle / \Lambda$ is suppressed by the new high energy scale $\Lambda$.

On the other hand, the $\Delta L=2$ washout processes become more significant for small $v_{\nu}[17,28]$. Notably, for a low scale seesaw mechanism, the narrow width condition $\Gamma_{1} / M_{1} \ll 1$ is satisfied. Therefore, the evolution of lepton asymmetry and DM abundance actually decouple from each other $[55,56]$. The evolution of abundance $Y_{N_{1}}$ and lepton asymmetry $Y_{\Delta L}$ is described by the Boltzmann equations

$$
\begin{aligned}
& \frac{d Y_{N_{1}}}{d z}=-D\left(Y_{N_{1}}-Y_{N_{1}}^{e q}\right), \\
& \frac{d Y_{\Delta L}}{d z}=-\epsilon_{1} D\left(Y_{N_{1}}-Y_{N_{1}}^{e q}\right)-W Y_{\Delta L} .
\end{aligned}
$$

The decay term is given by

$$
D=K z \frac{\mathcal{K}_{1}(z)}{\mathcal{K}_{2}(z)}
$$

For the washout term, two contributions are considered, i.e., $W=W_{I D}+W_{\Delta L=2}$, where the inverse decay term is

$$
W_{I D}=\frac{1}{4} K z^{3} \mathcal{K}_{1}(z)
$$

and the $\Delta L=2$ scattering term at low temperature is approximately [57]

$W_{\Delta L=2} \simeq \frac{0.186}{z^{2}}\left(\frac{246 \mathrm{GeV}}{v_{\nu}}\right)^{4}\left(\frac{M_{1}}{10^{10} \mathrm{GeV}}\right)\left(\frac{\bar{m}}{\mathrm{eV}}\right)^{2}$.

Here, $\bar{m}$ is the absolute neutrino mass scale, which is calculated as

$$
\bar{m}^{2}=m_{1}^{2}+m_{2}^{2}+m_{3}^{2}=3 m_{1}^{2}+\Delta m_{21}^{2}+\delta m_{31}^{2}
$$

for normal hierarchy. According to the latest global fit, we use the best fit values, i.e., $\Delta m_{21}^{2}=7.39 \times 10^{-5} \mathrm{eV}^{2}$ and $\delta m_{31}^{2}=$ $2.525 \times 10^{-3} \mathrm{eV}^{2}$ [58]. For tiny lightest neutrino mass $m_{1} \ll 10^{-2} \mathrm{eV}$, we actually have $\bar{m} \simeq \sqrt{\delta m_{31}^{2}} \sim 0.05 \mathrm{eV}$. Notably, the $\Delta L=2$ scattering term would be greatly enhanced when $v_{\nu} \ll v$, so this term is much more important than it is in vanilla leptogenesis. Then, the sphaleron processes convert the lepton asymmetry into baryon asymmetry as [59]

$$
Y_{\Delta B}=\frac{28}{79} Y_{\Delta(B-L)}=-\frac{28}{51} Y_{\Delta L}
$$

Figure 2 shows the washout effect of $\Delta L=2$ processes. In Fig. 2(a), a weak washout scenario is considered by fixing $K=10^{-2},\left|\epsilon_{1}\right|=10^{-6}, M_{1}=10^{6} \mathrm{GeV}$ while varying $v_{\nu}=10$, $1,0.1 \mathrm{GeV}$. It shows that for $v_{\nu}=10 \mathrm{GeV}$, the $\Delta L=2$ effect is not obvious, but for $v_{\nu}=1 \mathrm{GeV}$, the final baryon asymmetry $Y_{\Delta B}$ is diluted by over 3 orders of magnitude, while for $v_{\nu}=0.1 \mathrm{GeV}$, the $\Delta L=2$ effect is so strong that the final baryon asymmetry is negligible. The strong washout scenario with $K=10^{2},\left|\epsilon_{1}\right|=10^{-4}, M_{1}=10^{6} \mathrm{GeV}$ and varying $v_{\nu}=10,1,0.1 \mathrm{GeV}$ is illustrated in Fig. 2(b), where the final baryon asymmetry $Y_{\Delta B}$ for $v_{\nu}=1 \mathrm{GeV}$ is decreased by about 6 orders comparing with the case for $v_{\nu}=10 \mathrm{GeV}$. Therefore, the $\Delta L=2$ washout effects set a lower bound on $v_{\nu}$, i.e., $v_{\nu} \gtrsim 0.3 \mathrm{GeV}$ as suggested by Ref. [17]. Furthermore, since the $\Delta L=2$ washout term is also proportional to $M_{1}$, the larger $M_{1}$ is, the more obvious the washout effect is. The corresponding results are depicted in Fig. 2(c) for the weak washout and Fig. 2(d) for the strong washout. In this way, for a certain value of $v_{\nu}$, an upper bound on $M_{1}$ can be obtained. For instance, when $v_{\nu}=1 \mathrm{GeV}$, then $M_{1} \lesssim$ $10^{5} \mathrm{GeV}$ should be satisfied [28].

\section{DARK MATTER}

In our extension of the $\nu 2 \mathrm{HDM}$, the right-hand heavy neutrinos $N$ also couple with fermion singlet $\chi$ and scalar singlet $\phi$ via the Yukawa interaction. The complex Yukawa coupling coefficient $\lambda$ can lead to $C P$ violation in $N$ decay, and eventually produce asymmetric DM $\chi$ [55]. Instead, we consider another interesting scenario, i.e., the FIMP case with the real coupling $\lambda \ll 1[56] .{ }^{1}$ In this way, the interaction of DM $\chi$ is so weak that it never reaches thermalization. Its relic abundance is determined by the freeze-in mechanism [60], which is obtained by solving the following Boltzmann equation

$$
\frac{d Y_{\chi}}{d z}=D Y_{N_{1}} \mathrm{BR}_{\chi}
$$

where $\mathrm{BR}_{\chi}$ is the branching ratio of $N_{1} \rightarrow \chi \phi$. Because of the FIMP nature of $\chi$, the hierarchal condition $\mathrm{BR}_{\chi} \ll \mathrm{BR}_{\ell} \simeq 1$ is easily satisfied. The out-of-equilibrium condition for $N_{1} \rightarrow \chi \phi$ decay is $\Gamma_{\chi} / H(z=1) \simeq \mathrm{BR}_{\chi} \Gamma_{1} /$ $H(z=1)=\mathrm{BR}_{\chi} K<1$. In the following studies, we

\footnotetext{
${ }^{1}$ Since Ref. [56] considered canonical leptogenesis with only one scalar doublet, their leptogenesis framework is necessary at high scale. Thus, $\chi$ is from high scale $N$ in Ref. [56], and $\chi$ is from low scale $N$ in this paper. One can derive that $\lambda \simeq \sqrt{\mathrm{BR}_{\chi} K M_{1}} \times 1.2 \times 10^{-8}$. Therefore, the scale of $\lambda$ in a low scale seesaw mechanism is much smaller than in a canonical seesaw mechanism when $\mathrm{BR}_{\chi}$ and $K$ are of the same order.
} 



FIG. 2. The washout effect of $\Delta L=2$ processes. The cyan lines are the observed value $Y_{\Delta B}^{\text {obs }}=8.72 \times 10^{-11}$. (a) A weak washout scenario with varying $v_{\nu}$, (b) a strong washout scenario with varying $v_{\nu}$, (c) a weak washout scenario with varying $M_{1}$, and (d) a strong washout scenario with varying $M_{1}$.

mainly take $\mathrm{BR}_{\chi}<10^{-2}$ and $K \lesssim 10$; thus, the out-ofequilibrium condition is always satisfied. According to the above Boltzmann equation, we can estimate the asymptotic abundance of $\chi$ as [56]

$$
Y_{\chi}(\infty) \simeq Y_{N_{1}}(0) \mathrm{BR}_{\chi}\left(1+\frac{15 \pi \zeta(5)}{16 \zeta(3)} K\right)
$$

Then, the corresponding relic abundance is

$$
\begin{aligned}
\Omega_{\chi} h^{2} & =\frac{m_{\chi} s_{0} Y_{\chi}(\infty)}{\rho_{c}} h^{2} \\
& \simeq 0.12 \times\left(\frac{m_{\chi}}{\mathrm{keV}}\right)\left(\frac{\mathrm{BR}_{\chi}}{10^{-3}}\right)\left(0.009+\frac{K}{44}\right),
\end{aligned}
$$

where $s_{0}=2891.2 \mathrm{~cm}^{-3}, \rho_{c}=1.05371 \times 10^{-5} h^{2} \mathrm{GeV} \mathrm{cm}^{-3}$ [61]. Typically, the observed relic abundance can be obtained with $m_{\chi} \sim 4 \mathrm{keV}, \mathrm{BR}_{\chi} \sim 10^{-3}$, and $K \sim 10$. The evolution of DM abundances is shown in Fig. 3. It is clear that when the temperature goes down to $z=m_{\chi} / T \sim 5$, the abundances $Y_{\chi}$ freeze in and keep at a constant. The left panel of Fig. 3 indicates that $m_{\chi}$ is inversely proportional to $\mathrm{BR}_{\chi}$ when the decay parameter $K$ is a constant. For instance, sub-MeV-scale light DM is obtained when $\mathrm{BR}_{\chi}>10^{-6}$ with $K=10$. The right panel of Fig. 3 shows the impact of decay parameter $K$. Affected by the constant term before $K$ in Eq. (42), we can only conclude that the smaller the $K$ is, the larger the $m_{\chi}$ is. Besides, we also find that the discrepancy between the numerical and analytical results of $Y_{\chi}(\infty)$ increases when $K$ decreases. Therefore, we adopt the numerical result of $Y_{\chi}(\infty)$ for a more precise calculation in the following discussion. 

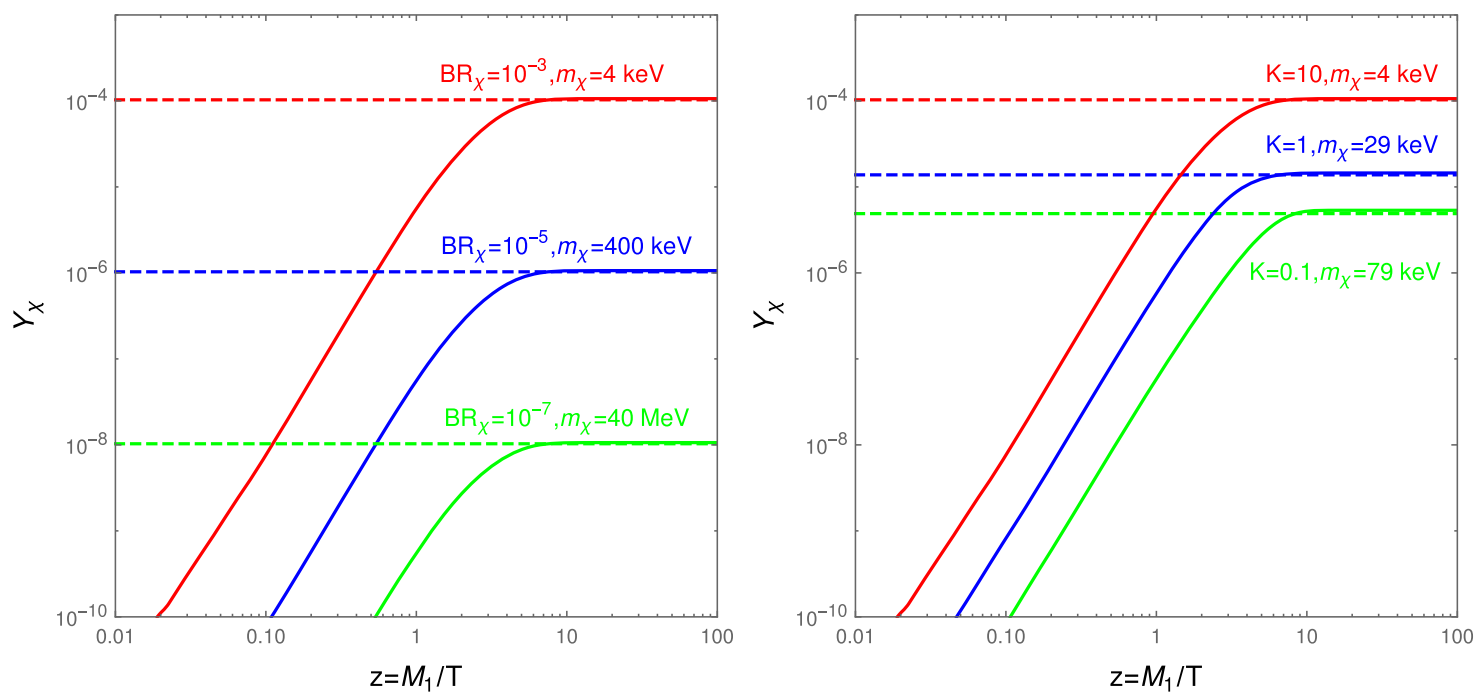

FIG. 3. Evolution of dark matter abundance with parameter $z=M_{1} / T$. We fix $K=10$ in the left panel and $\mathrm{BR}_{\chi}=10^{-3}$ in the right panel. The dashed horizontal lines correspond to the estimated results with Eq. (41). DM mass $m_{\chi}$ is obtained by setting $\Omega_{\chi} h^{2}=0.12$ with the numerical results of $Y_{\chi}(\infty)$.

The dominant constraint on FIMP DM $\chi$ comes from its free-streaming length, which describes the average distance a particle travels without a collision [56]

$r_{F S}=\int_{a_{r h}}^{a_{e q}} \frac{\langle v\rangle}{a^{2} H} d a \approx \frac{a_{\mathrm{NR}}}{H_{0} \sqrt{\Omega_{R}}}\left(0.62+\ln \left(\frac{a_{e q}}{a_{\mathrm{NR}}}\right)\right)$,

where $\langle v\rangle$ is the averaged velocity of $\mathrm{DM} \chi$, and $a_{e q}$ and $a_{r h}$ represent scale factors in equilibrium and reheating, respectively. We use the results $H_{0}=67.3 \mathrm{~km} \mathrm{~s}^{-1} \mathrm{Mpc}^{-1}$, $\Omega_{R}=9.3 \times 10^{-5}$, and $a_{e q}=2.9 \times 10^{-4}$ obtained from Ref. [62]. The nonrelativistic scale factor for FIMP DM is

$$
a_{\mathrm{NR}}=\frac{T_{0}}{2 m_{\chi}}\left(\frac{g_{*, 0}}{g_{*, r h}}\right)^{\frac{1}{3}} K^{-\frac{1}{2}} .
$$

Taking $g_{*, 0}=3.91, \quad g_{*, r h}=106.75, \quad$ and $T_{0}=2.35 \times$ $10^{-4} \mathrm{eV}$, finally we can get

$$
\begin{aligned}
r_{F S} \simeq & 2.8 \times 10^{-2}\left(\frac{\mathrm{keV}}{m_{\chi}}\right)\left(\frac{50}{K}\right)^{\frac{1}{2}} \\
& \times\left(1+0.09 \ln \left[\left(\frac{m_{\chi}}{\mathrm{keV}}\right)\left(\frac{K}{50}\right)^{\frac{1}{2}}\right]\right) \mathrm{Mpc} .
\end{aligned}
$$

The most stringent bound on $r_{F S}$ comes from small structure formation $r_{F S}<0.1 \mathrm{Mpc}$ [63]. The relationship between the mass of $\chi$ and its free-streaming length is depicted in Fig. 4. Basically speaking, warm DM is obtained for $m_{\chi} \sim 10 \mathrm{keV}$ while $K \in[0.01,100]$.
Meanwhile, $\chi$ becomes cold DM when $\chi$ is sufficiently heavy and/or the decay parameter $K$ is large enough.

\section{COMBINED ANALYSIS}

After studying some benchmark points, it would be better to figure out the viable parameter space for successful leptogenesis and DM. We then perform a random scan over the following parameter space:

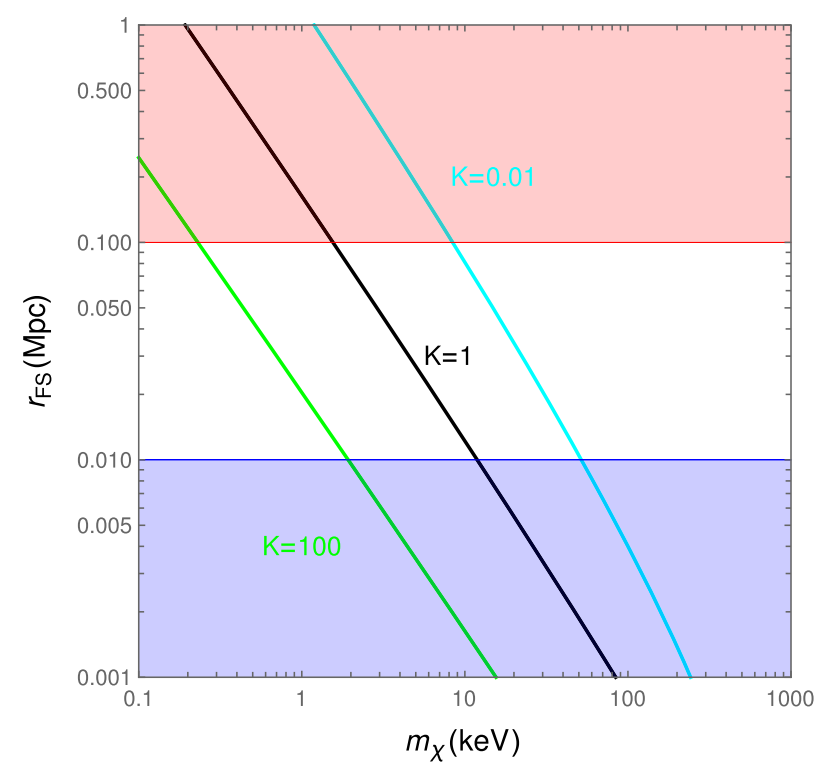

FIG. 4. Influence of free streaming on DM mass. The red area $\left(r_{F S}>0.1 \mathrm{Mpc}\right)$, white area $\left(0.1 \mathrm{Mpc}>r_{F S}>0.01 \mathrm{Mpc}\right)$, and blue area $\left(r_{F S}<0.01 \mathrm{Mpc}\right)$ correspond to the hot, warm, and cold DM scenario [64], respectively. 

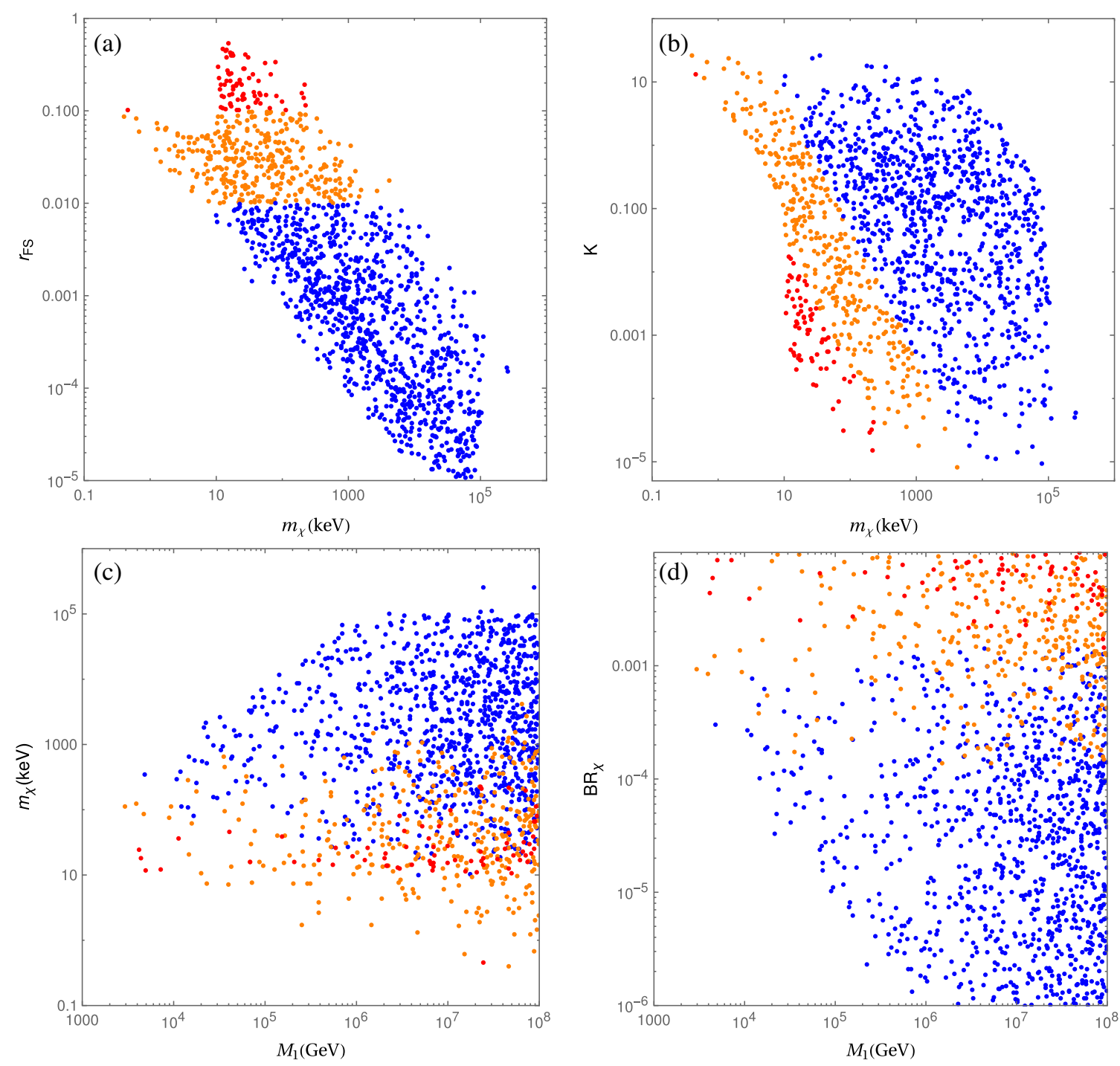

FIG. 5. Viable parameter space for DM. The red, orange, and blue points correspond to hot, warm, and cold DM, respectively.

$$
\begin{aligned}
m_{1} & \in\left[10^{-12}, 10^{-2}\right] \mathrm{eV}, \quad M_{1} \in\left[10^{3}, 10^{8}\right] \mathrm{GeV}, \\
v_{\nu} & \in\left[10^{-2}, 10^{2}\right] \mathrm{GeV}, \\
\operatorname{Re}\left(\omega_{12,13,23}\right) & \in\left[10^{-10}, 1\right], \quad \operatorname{Im}\left(\omega_{12,13,23}\right) \in\left[10^{-10}, 1\right], \\
\mathrm{BR}_{\chi} & \in\left[10^{-6}, 10^{-2}\right] .
\end{aligned}
$$

During the scan, we have fixed $M_{2} / M_{1}=M_{3} / M_{2}=10$. The final obtained baryon asymmetry $Y_{\Delta B}$ is required to be within $3 \sigma$ ranges of the observed value, i.e., $Y_{\Delta B} \in[8.60,8.84] \times 10^{-11}$. The results are shown in Figs. 5 and 6 for DM and leptogenesis, respectively.

Let us consider the DM results in Fig. 5 first. According to the dominant constraint from free-streaming length $r_{F S}$, we can divide viable samples into three scenarios in Fig. 5(a). Of course, the hot DM scenario is not favored by small structure formation. For warm DM, $m_{\chi} \in$ $\left[0.3,2 \times 10^{3}\right] \mathrm{keV}$ is possible. Meanwhile for cold DM, $m_{\chi} \in\left[10,2 \times 10^{5}\right] \mathrm{keV}$ is allowed. And $r_{F S}$ is down to about $10^{-5} \mathrm{Mpc}$ when $m_{\chi} \sim 10^{5} \mathrm{keV}$. From Fig. 5(b), we are aware that the hot DM samples correspond to those with small DM mass $m_{\chi}$ and very weak washout effect $K \lesssim 10^{-2}$. Figure 5(c) shows the samples in the $m_{\chi}-M_{1}$ plane. Three kinds of DM are all possible for a certain value of $M_{1}$. By the way, it is interesting to obtain an upper limit on $m_{\chi}$ when $M_{1} \lesssim 10^{6} \mathrm{GeV}$. This indicates that for $\mathrm{TeV}$-scale leptogenesis, FIMP DM should be keV to sub$\mathrm{MeV}$. The result for $\mathrm{BR}_{\chi}$ is shown in Fig. 5(d), which tells us that warm $\mathrm{DM}$ requires $\mathrm{BR}_{\chi} \gtrsim 10^{-4}$ and cold $\mathrm{DM}$ requires $\mathrm{BR}_{\chi} \lesssim 10^{-3}$, respectively.

Then, we consider the leptogenesis results in Fig. 6 . The generalized Davidson-Ibarra bound is clearly seen in Fig. 6(a). The (warm and cold DM) allowed samples show that the mass of $N_{1}$ for successful leptogenesis could be down to about $3 \mathrm{TeV}$. The viable region of the $v_{\nu}-M_{1}$ 

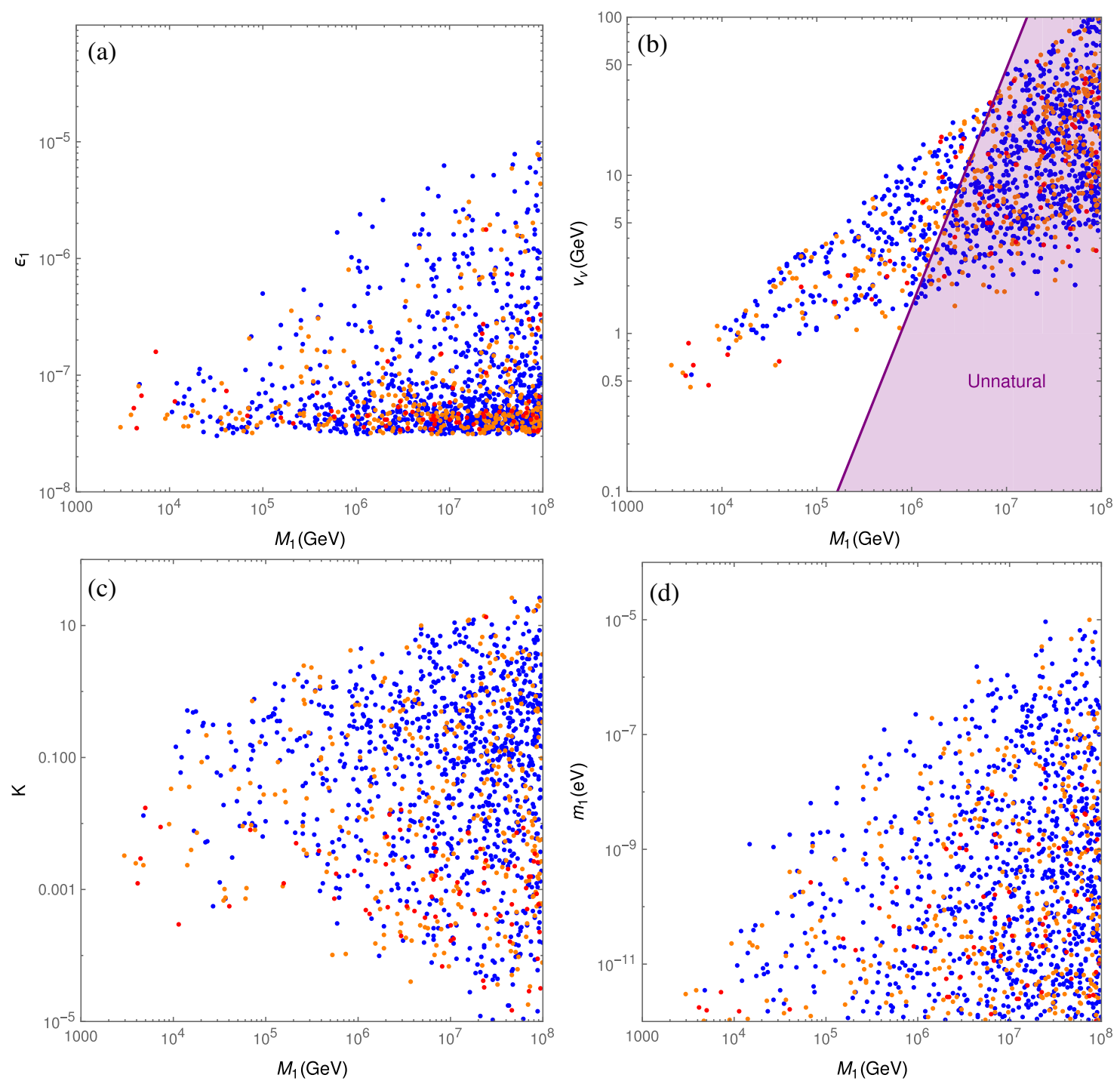

FIG. 6. Same as Fig. 5, but for leptogenesis.

plane is shown in Fig. 6(b), which is consistent with the theoretical bounds discussed in Ref. [17]. For completeness, the naturalness bound in Eq. (4) is also shown. Therefore, natural leptogenesis is viable for $3 \times 10^{3} \mathrm{GeV} \lesssim M_{1} \lesssim 7 \times$ $10^{6} \mathrm{GeV}$ with $0.4 \mathrm{GeV} \lesssim v_{\nu} \lesssim 30 \mathrm{GeV}$. The result for decay parameter $K$ is given in Fig. 6(c), which shows that $K \lesssim 10$ should be satisfied when $M_{1} \lesssim 10^{8} \mathrm{GeV}$. Actually, for $M_{1} \lesssim 10^{5} \mathrm{GeV}$, all the samples are within the weak washout region. An upper bound on lightest neutrino mass $m_{1}$ is clearly seen in Fig. 6(d). It also indicates that successful leptogenesis in the $\nu 2 \mathrm{HDM}$ requires that $m_{1}$ must be extremely tiny, e.g., $m_{1} \lesssim 10^{-11} \mathrm{eV}$ for $M_{1} \sim 10^{4} \mathrm{GeV}$.

Before ending this section, we give a brief discussion on the collider signature. According to the results of leptogenesis in Fig. 6, not too small $v_{\nu}$ is favored. In such a scenario, the branching ratios of neutrinophilic scalars are quite different from the scenario with small $v_{\nu}$ [45,65-67], but are similar to type-I 2HDM [68]. Currently, if $m_{\Phi_{\nu}}$ is smaller than $m_{t}$, the most stringent constraint comes from $t \rightarrow b H^{ \pm}\left(H^{ \pm} \rightarrow \tau^{ \pm} \nu\right)$ [69], which could exclude the region $v_{\nu} \gtrsim 18 \mathrm{GeV}$ [70]. Meanwhile, if $m_{Z}+m_{h} \lesssim m_{\phi_{\nu}} \lesssim 2 m_{t}$, the channel $A \rightarrow Z h(h \rightarrow b \bar{b})$ could exclude the region $v_{\nu} \gtrsim 24 \mathrm{GeV}$ [71]. For heavier additional scalars with $m_{\Phi_{\nu}}>2 m_{t}$, the signature $A / H \rightarrow t \bar{t}$ is only able to probe the region $v_{\nu} \gtrsim 174 \mathrm{GeV}[72,73]$. Therefore, the experimental bounds on neutrinophilic scalars can be easily escaped providing $m_{\Phi_{\nu}}$ is large enough. At the HL LHC, the signature $A \rightarrow Z h(h \rightarrow b \bar{b})$ would reach $v_{\nu} \sim 10 \mathrm{GeV}$ [73]. Then the observation of this signature will indicate $M_{1} \sim 10^{6} \mathrm{GeV}$ and $m_{1} \lesssim 10^{-7} \mathrm{eV}$.

\section{CONCLUSION}

In this paper, we propose an extended $\nu 2 \mathrm{HDM}$ to interpret the neutrino mass, leptogenesis, and dark matter simultaneously. This model contains one neutrinophilic 
scalar doublet $\Phi_{\nu}$, three right-hand heavy neutrinos $N$, which account for low scale neutrino mass generation similar to the type-I seesaw mechanism. Leptogenesis is generated due to the $C P$-violating decays of right-hand neutrino $N \rightarrow \ell_{L} \Phi_{\nu}^{*}$, $\bar{\ell}_{L} \Phi_{\nu}$. The dark sector contains one scalar singlet $\phi$ and one Dirac fermion singlet $\chi$, which are charged under a $Z_{2}$ symmetry. Provided $m_{\chi}<m_{\phi}$ and $\lambda \ll 1, \chi$ is a FIMP DM candidate within this paper. The relic abundance of $\chi$ is produced by $N \rightarrow \chi \phi$. Therefore, we have a common origin, i.e., the heavy right-hand neutrinos $N$, for tiny neutrino mass, baryon asymmetry, and dark matter.

In the framework of $\nu 2 \mathrm{HDM}$, the asymmetry $\epsilon_{1}$ and decay parameter $K$ are both enhanced by the smallness of $v_{\nu}$. By explicit calculation, we show that the decay parameter $K$ can be suppressed under certain circumstances. And a hierarchical structure of Yukawa coupling $y$ is needed to induce leptogenesis at low scale for the nondegenerate mass of $N$. The importance of the $\Delta L=2$ washout process is also illustrated. As for FIMP DM, the relic abundance mainly depends on the branching ratio $\mathrm{BR}_{\chi}$ and decay parameter $K$, and $m_{\chi}$ is typically at the order of $\mathrm{keV}$ to $\mathrm{MeV}$ scale. Meanwhile, the freestreaming length sets a stringent bound. The viable parameter space for successful leptogenesis and DM is obtained by solving the corresponding Boltzmann equations. To keep this model natural, we find $10^{3} \mathrm{GeV} \lesssim$ $M_{1} \lesssim 10^{6} \mathrm{GeV}, 0.4 \mathrm{GeV} \lesssim v_{\nu} \lesssim 30 \mathrm{GeV}, m_{1} \lesssim 10^{-5} \mathrm{eV}$, and $K \lesssim 10$ is favored by leptogenesis. Meanwhile, the warm (cold) DM mass in the range $m_{\chi} \in\left[0.3,2 \times 10^{3}\right] \mathrm{keV}$ $\left(m_{\chi} \in\left[10,2 \times 10^{5}\right] \mathrm{keV}\right)$ is predicted with $\mathrm{BR}_{\chi} \gtrsim 10^{-4}$ $\left(\mathrm{BR}_{\chi} \lesssim 10^{-3}\right)$.

\section{ACKNOWLEDGMENTS}

This work is supported by the National Natural Science Foundation of China under Grant No. 11805081 and the Natural Science Foundation of Shandong Province under Grants No. ZR2019QA021 and No. ZR2018MA047.
[1] Y. Fukuda et al. (Super-Kamiokande Collaboration), Phys. Rev. Lett. 81, 1562 (1998).

[2] Q. R. Ahmad et al. (SNO Collaboration), Phys. Rev. Lett. 89, 011301 (2002).

[3] P. Minkowski, Phys. Lett. 67B, 421 (1977).

[4] R. N. Mohapatra and G. Senjanovic, Phys. Rev. Lett. 44, 912 (1980).

[5] M. Fukugita and T. Yanagida, Phys. Lett. B 174, 45 (1986).

[6] S. Davidson and A. Ibarra, Phys. Lett. B 535, 25 (2002).

[7] F. Vissani, Phys. Rev. D 57, 7027 (1998).

[8] J. D. Clarke, R. Foot, and R. R. Volkas, Phys. Rev. D 91, 073009 (2015).

[9] A. Pilaftsis and T. E. J. Underwood, Nucl. Phys. B692, 303 (2004).

[10] E. K. Akhmedov, V. A. Rubakov, and A. Y. Smirnov, Phys. Rev. Lett. 81, 1359 (1998).

[11] T. Asaka and M. Shaposhnikov, Phys. Lett. B 620, 17 (2005).

[12] T. Hambye and D. Teresi, Phys. Rev. Lett. 117, 091801 (2016).

[13] T. Hambye and D. Teresi, Phys. Rev. D 96, 015031 (2017).

[14] S. Baumholzer, V. Brdar, and P. Schwaller, J. High Energy Phys. 08 (2018) 067.

[15] T. Alanne, T. Hugle, M. Platscher, and K. Schmitz, J. Cosmol. Astropart. Phys. 03 (2019) 037.

[16] W. Chao and M. J. Ramsey-Musolf, Phys. Rev. D 89, 033007 (2014).

[17] J. D. Clarke, R. Foot, and R. R. Volkas, Phys. Rev. D 92 , 033006 (2015).

[18] E. Ma, Mod. Phys. Lett. A 21, 1777 (2006).

[19] T. Hambye, F. Ling, L. Lopez Honorez, and J. Rocher, J. High Energy Phys. 07 (2009) 090.
[20] S. Kashiwase and D. Suematsu, Phys. Rev. D 86, 053001 (2012).

[21] S. Kashiwase and D. Suematsu, Eur. Phys. J. C 73, 2484 (2013).

[22] J. Racker, J. Cosmol. Astropart. Phys. 03 (2014) 025.

[23] T. Hugle, M. Platscher, and K. Schmitz, Phys. Rev. D 98 , 023020 (2018).

[24] D. Borah, A. Dasgupta, and S. K. Kang, arXiv:1806.04689.

[25] D. Borah, P. S. B. Dev, and A. Kumar, Phys. Rev. D 99 , 055012 (2019).

[26] D. Mahanta and D. Borah, J. Cosmol. Astropart. Phys. 04 (2020) 032.

[27] E. Ma, Phys. Rev. Lett. 86, 2502 (2001).

[28] N. Haba and O. Seto, Prog. Theor. Phys. 125, 1155 (2011).

[29] N. Haba and O. Seto, Phys. Rev. D 84, 103524 (2011).

[30] S. Dodelson and L. M. Widrow, Phys. Rev. Lett. 72, 17 (1994).

[31] M. Drewes et al., J. Cosmol. Astropart. Phys. 01 (2017) 025.

[32] A. Adulpravitchai and M. A. Schmidt, J. High Energy Phys. 12 (2015) 023.

[33] Z. L. Han, B. Zhu, L. Bian, and R. Ding, arXiv:1812.00637.

[34] A. Boyarsky, M. Drewes, T. Lasserre, S. Mertens, and O. Ruchayskiy, Prog. Part. Nucl. Phys. 104, 1 (2019).

[35] S. Antusch, P. Di Bari, D. A. Jones, and S. F. King, Phys. Rev. D 86, 023516 (2012).

[36] D. Mahanta and D. Borah, J. Cosmol. Astropart. Phys. 11 (2019) 021.

[37] M. Chianese and S. F. King, J. Cosmol. Astropart. Phys. 09 (2018) 027.

[38] M. Chianese, B. Fu, and S. F. King, J. Cosmol. Astropart. Phys. 03 (2020) 030. 
[39] E. Aprile et al. (XENON Collaboration), Phys. Rev. Lett. 121, 111302 (2018).

[40] M. Ackermann et al. (Fermi-LAT Collaboration), Phys. Rev. Lett. 115, 231301 (2015).

[41] N. Bernal, M. Heikinheimo, T. Tenkanen, K. Tuominen, and V. Vaskonen, Int. J. Mod. Phys. A 32, 1730023 (2017).

[42] S. M. Davidson and H. E. Logan, Phys. Rev. D 80, 095008 (2009).

[43] T. Morozumi, H. Takata, and K. Tamai, Phys. Rev. D 85, 055002 (2012); 89, 079901(E) (2014).

[44] N. Haba and T. Horita, Phys. Lett. B 705, 98 (2011).

[45] C. Guo, S. Y. Guo, Z. L. Han, B. Li, and Y. Liao, J. High Energy Phys. 04 (2017) 065.

[46] G. Aad et al. (ATLAS Collaboration), Phys. Lett. B 716, 1 (2012).

[47] S. Chatrchyan et al. (CMS Collaboration), Phys. Lett. B 716, 30 (2012).

[48] P. A. N. Machado, Y. F. Perez, O. Sumensari, Z. Tabrizi, and R. Z. Funchal, J. High Energy Phys. 12 (2015) 160.

[49] J. A. Casas and A. Ibarra, Nucl. Phys. B618, 171 (2001).

[50] A. Ibarra and G. G. Ross, Phys. Lett. B 591, 285 (2004).

[51] E. Nardi, Y. Nir, E. Roulet, and J. Racker, J. High Energy Phys. 01 (2006) 164.

[52] S. Davidson, E. Nardi, and Y. Nir, Phys. Rep. 466, 105 (2008).

[53] N. Aghanim et al. (Planck Collaboration), arXiv:1807 .06209 .

[54] W. Wang and Z. L. Han, J. High Energy Phys. 04 (2017) 166.

[55] A. Falkowski, J. T. Ruderman, and T. Volansky, J. High Energy Phys. 05 (2011) 106.

[56] A. Falkowski, E. Kuflik, N. Levi, and T. Volansky, Phys. Rev. D 99, 015022 (2019).
[57] W. Buchmuller, P. Di Bari, and M. Plumacher, Ann. Phys. (Amsterdam) 315, 305 (2005).

[58] I. Esteban, M. C. Gonzalez-Garcia, A. HernandezCabezudo, M. Maltoni, and T. Schwetz, J. High Energy Phys. 01 (2019) 106.

[59] J. A. Harvey and M. S. Turner, Phys. Rev. D 42, 3344 (1990).

[60] L. J. Hall, K. Jedamzik, J. March-Russell, and S. M. West, J. High Energy Phys. 03 (2010) 080.

[61] M. Tanabashi et al. (Particle Data Group), Phys. Rev. D 98, 030001 (2018).

[62] P. A. R. Ade et al. (Planck Collaboration), Astron. Astrophys. 594, A13 (2016).

[63] A. Berlin and N. Blinov, Phys. Rev. Lett. 120, 021801 (2018).

[64] A. Merle, V. Niro, and D. Schmidt, J. Cosmol. Astropart. Phys. 03 (2014) 028.

[65] N. Haba and K. Tsumura, J. High Energy Phys. 06 (2011) 068.

[66] W. Wang and Z. L. Han, Phys. Rev. D 94, 053015 (2016).

[67] K. Huitu, T. J. Karkkainen, S. Mondal, and S. K. Rai, Phys. Rev. D 97, 035026 (2018).

[68] G. C. Branco, P. M. Ferreira, L. Lavoura, M. N. Rebelo, M. Sher, and J. P. Silva, Phys. Rep. 516, 1 (2012).

[69] A. M. Sirunyan et al. (CMS Collaboration), J. High Energy Phys. 07 (2019) 142.

[70] P. Sanyal, Eur. Phys. J. C 79, 913 (2019).

[71] M. Aaboud et al. (ATLAS Collaboration), J. High Energy Phys. 03 (2018) 174; 11 (2018) 051(E).

[72] M. Aaboud et al. (ATLAS Collaboration), Phys. Rev. Lett. 119, 191803 (2017)

[73] N. Chen, T. Han, S. Li, S. Su, W. Su, and Y. Wu, arXiv: 1912.01431. 